\title{
LA PERCEPCIÓN DEL TRATAMIENTO DE LOS MEDIOS DE COMUNICACIÓN HACIA LOS TEMAS RELACIONADOS CON LA COMUNIDAD GLBTI
}

\section{THE PERCEPTION OF THE TREATMENT OF THE MASS MEDIA OVER ISSUES LINKED TO THE TGLBI COMMUNITY}

\section{FERNANDA CADENA MELO ${ }^{1}$ FRANCISCO PACHECO SEMPÉRTEGUI ${ }^{2}$}

Recibido: 3 de enero de 2017 Aceptado: 6 de marzo de 2017

\footnotetext{
${ }^{1}$ Pontificia Universidad Católica del Ecuador, Facultad de Comunicación Lingüística y Literatura, Escuela de Comunicación, Quito, Ecuador (fernandapaulinac@gmail.com).

${ }^{2}$ Pontificia Universidad Católica del Ecuador, Facultad de Comunicación Lingüística y Literatura, Escuela de Comunicación, Quito, Ecuador (fapacheco@puce.edu.ec).
} 



\section{LA PERCEPCIÓN DEL TRATAMIENTO DE LOS MEDIOS DE COMUNICACIÓN HACIA LOS TEMAS RELACIONADOS CON LA COMUNIDAD GLBTI}

\section{THE PERCEPTION OF THE TREATMENT OF THE MASS MEDIA OVER ISSUES LINKED TO THE TGLBI COMMUNITY}

FERNANDA CADENA MELO, FRANCISCO PACHECO SEMPÉRTEGUI

PALABRAS CLAVE: cambio social, GLBTI, medios de comunicación, percepción

KEYWORDS: social change, TGLBI, mass media, perception

\section{RESUMEN}

El objetivo general de esta investigación es conocer la percepción de la sociedad, específicamente de los jóvenes, sobre el tratamiento que los medios de comunicación dan a los temas relacionados con la comunidad GLBTI. La población investigada comprende dos institutos de educación superior privados de la ciudad de Quito: la Pontificia
Universidad Católica del Ecuador (PUCE) y a la Universidad Politécnica Salesiana (UPS). Se eligió trabajar con cuestionarios para realizar un sondeo de opinión. Se determinó la aplicación de 161 encuestas útiles para análisis según conceptos del muestreo no probabilístico. El público encuestado reflejó una falta de conocimiento generalizado y percepciones 
vagas sobre conceptos que se refieren a la diversidad sexual. El público percibe que los medios de comunicación tradi- cionales en el país emiten material informativo escaso sobre diversidad sexual y derechos de personas GLBTI.

\section{ABSTRACT}

The main objective of this research is to know the perception of the society, specifically of young people, on the treatment that the mass media give to the issues related to the LGBTI community. The research population include two main universities in Quito: The Pontificia Universidad Católica del EcuadorPUCE and the Universidad Politécnica Salesiana - UPS. We worked with ques- tionnaires to conduct an opinion poll. We applied 161 surveys for the analysis, according to concepts of non-probabilistic sampling. The surveyed public reflected a lack of knowledge and vague perceptions about concepts linked to sexual diversity. The public noticed that the traditional media in the country show limited information about sexual diversity and LGBTI rights.

\section{INTRODUCCIÓN}

La segregación de la comunidad GLBTI' se traduce en una coyuntura problemática, ya que la cotidianidad con la que se producen manifestaciones discriminatorias entorpece el reconocimiento de derechos civiles que, en teoría, amparan a todos los sujetos. En Ecuador, en el plano legislativo se han tomado medidas para frenar actitudes cotidianas que, a manera de rechazo o burla, distorsionan la realidad sobre la diversidad sexual, por ejemplo las sanciones que se contemplan en la Ley Orgánica de Co- municación al transmitir contenido que abarque este tema. Aun así, el colectivo GLBTI impulsa una lucha constante pues existen derechos que para ellos son reconocidos parcialmente y por lo que se consideran vulnerados. Como el caso de la aprobación de la unión de hecho entre personas del mismo sexo, mas no el matrimonio entre ellas o la adopción por parte de estas parejas.

Debido a la incidencia de la comunicación en el imaginario social, esta podría actuar como gestora de un cam-

\footnotetext{
${ }^{1}$ GLBTI: Gays, Lesbianas, Bisexuales, Transgénero, Transexuales, Travestis e Intersexuales.
} 
bio social en favor de la desmitificación de estereotipos sobre la sexualidad.

Este trabajo comprende un estudio de percepción realizado mediante una investigación exploratoria y cuyo análisis de datos proporcionó una aproximación a los conocimientos, actitudes y prácticas de la sociedad quiteña, específicamente de los jóvenes, hacia la comunidad GLBTI.

Entonces, el objetivo general de esta investigación es conocer la percepción de la sociedad, sobre todo jóvenes, acerca del tratamiento que los medios de comunicación dan a los temas relacionados con la comunidad GLBTI. Para ello se plantean los siguientes objetivos específicos:

a. Determinar el nivel de conocimiento sobre la sigla GLBTI.

b. Conocer las actitudes cotidianas que una población de jóvenes tiene frente a la comunidad GLBTI

c. Identificar la impresión que tienen los jóvenes sobre el tratamiento de los medios de comunicación hacia la comunidad GLBTI.

\section{COMUNICACIÓN Y CAMBIO SOCIAL}

\section{Introducción}

La comunicación como forma de interacción entre individuos no es exclusivamente humana, ya que el intercambio de estímulos simples al que responden formas de vida menos evolucionadas también se considera como un acto comunicativo (De Fleur, 1972). Entonces, no podemos remitirnos únicamente a un concepto clásico como "un proceso en virtud del cual A envía un mensaje a B, que provoca un efecto" (O'Sullivan, Hartley, Saunders, Montgomery, \& Fiske, 1997, p. 66).

Desde la comunicación más primitiva y gestual hasta la interacción digital, todas las sociedades han encontrado formas para comunicarse, ya que es impensable que un grupo viva y se organice sin comunicar o comunicarse. Las condiciones actuales en las que se desarrolla la comunicación son únicas debido a que nos enfrentamos a una revolución tecnológica permanente, que parece romper barreras de espacio y tiempo. Nos hemos acostumbrado a vivir dentro de un proceso de cambio al que aceptamos y nos vemos forzados a adaptarnos de forma acelerada para sobrevivir en este mundo de una vertiginosa tecnología que nos abruma.

Día a día somos bombardeados con todo tipo de información que influye de forma voluntaria o involuntaria en 
nuestras acciones y relaciones de grupo. Al mismo tiempo, tenemos la ventaja de la globalización, en la medida que tenemos el poder y la capacidad de acceder a una cantidad de datos mayor a la que nunca antes fue posible gracias a distintos medios tecnológicos, canales y recursos. En consecuencia, la cultura misma sufre transformaciones aceleradas. Vivimos la apropiación de lo global mientras se produce una aculturación local. Con esto nos referimos a que los procesos, incluso los comunicativos, son pensados en función de las masas con el fin de lograr imponer tendencias de cultura generales. Por ejemplo, la comunicación utilizada por la publicidad para mantener una sociedad de consumo masivo.

Actualmente, la comunicación desde un enfoque crítico toma en cuenta tanto los procesos como los contextos culturales e históricos que influyen en el acto comunicativo (Fernández Collado \& Galguera García, 2008). Es decir, hablamos de una realidad comunicativa contextualizada y única que influye en cada elemento de la comunicación.

\section{Comunicación para el cambio social}

La acción comunicativa nos resulta tan familiar como acción cotidiana, que como concepto, pasa casi desapercibida. Y aunque todos somos participantes activos del proceso comunicati- vo en la sociedad, no todos la utilizamos con el mismo enfoque ni con los mismos propósitos.

Se ha visto a la comunicación como un campo relegado, ya que algunos sectores la han dejado a un lado minimizándola, sin tomar en cuenta el poder de interacción, diálogo y acuerdos a los que se podría llegar mediante ella. Esta puede actuar como un medio de poder influyente, por lo que gestionada correctamente se la puede entender como herramienta de avance social.

Paradójicamente, los medios masivos de comunicación, así como los líderes de opinión han establecido y reforzado ideas y símbolos mediante diferentes manifestaciones a través de los años. Debido a que gozan de credibilidad como fuente verídica y replicable de información ahora resultan corresponsables de legitimar estereotipos obsoletos que perjudican o entorpecen el progreso de la sociedad.

En este punto es preciso mencionar a las ideas como imaginarios sociales que se forjan. Y junto a ello, no podemos negar la transcendencia que los medios masivos de comunicación han tenido a lo largo de la historia para la construcción y consolidación de juicios. Pero ¿cómo se aprenden estas ideas? Se puede decir que los procesos que llevan a estereotipar a los individuos o cosas inician con la categorización. 
La categorización social cumple la función de economizar esfuerzos que el sistema cognitivo debe hacer a la hora de procesar la información que nos proporcionan los estímulos exteriores, y facilita la orientación de la persona en el mundo. Según Garrido y Álvaro (2007) es el proceso inductivo, que hace posible la identificación de un objeto a partir de una información insuficiente, y el proceso deductivo, mediante el cual se atribuyen a un objeto o a un acontecimiento las propiedades de la categoría a la que pertenece.

Desde este punto de vista habría una explicación que a nivel cognitivo refuerza las ideas de forma inconsciente, pero estas convenciones son aprendidas por la persona mediante el contacto con determinados ambientes; además, si el contacto es superficial se puede obtener datos pobres sobre ciertos contextos. Esto es lo que ocurriría con los grupos sociales relegados, ya que por la convivencia restringida de otros miembros de la sociedad con ellos, se crean o mantiene ideas frívolas que luego son popularizadas entre grupos.

Cuando estos juicios se refieren a la sexualidad de las personas están relacionados con los roles tradicionales de sexo establecidos por la sociedad. Hablar de género en lugar de sexo podría causar desconcierto ya que asignar roles a los géneros es más complejo que hablar de los papeles establecidos para hombre y mujer. Estaríamos enfrentando los conceptos de lo femenino y masculino que rebasan a la idea del sexo remitiéndonos solo a los órganos genitales de los individuos. Para romper con la categorización social se necesitaría el apoyo de un proceso, en este caso la comunicación enfocada hacia el cambio social.

Alfonso Gumucio (2004, p. 4) señala
que la comunicación para el cambio
social: [E]s una comunicación ética, es decir, de la identidad y de la afirmación de valores; amplifica las voces ocultas o negadas, y busca potenciar su presencia en la esfera pública. Recupera el diálogo y la participación como ejes centrales; ambos elementos existían entrelazados con otros modelos y paradigmas y estaban presentes en la teoría como en un gran número de experiencias concretas, pero no tenían carta de ciudadanía entre los modelos dominantes, de modo que no alimentaron suficientemente la reflexión.

Desde esta perspectiva, la función de este tipo de comunicación es buscar un cambio social que conlleve un progreso. Su meta es devolver a la esfera social a los actores que hayan 
sido relegados por la misma sociedad, mediante una manifestación libre pero responsable de sí mismos. De esta manera, la expresión y consolidación de una identidad daría la oportunidad de participación activa y equitativa de distintos sectores sociales.

La comunicación orientada hacia la transformación es un reto para los comunicadores, ya que el enfoque debe atravesar los medios tradicionales de comunicación como a organizaciones, a los sectores gubernamentales y a la sociedad civil pues representa una reorganización y apertura hacia procesos comunicativos que deben abarcar la ruptura de paradigmas que buscan mantener el statu quo. La innovación en los mensajes, la construcción del contenido y el sentido que se le debe dar así como, el uso adecuado de la información, la divulgación por canales alternativos, la visibilización de grupos minoritarios, el apoyo a las causas sociales; podrían contar como elementos de soporte para el cambio mediante el trabajo de la comunicación sobre las ideas enraizadas a la sociedad.

Gumucio (2004) sostiene que la importancia del proceso más simple de la comunicación es el diálogo. Esto nos hace reflexionar sobre cómo se toma a este modo de interacción, porque en realidad actuaría como un pilar de investigación y acuerdos con los grupos de interés. Pues resultaría ser la forma más sencilla de conocer los objetivos que individuos y agrupaciones buscan. Desde este primer acercamiento se podría pensar en explorar en una escala mayor que valide el despliegue de recursos y procesos para afrontar una situación conflictiva.

Lo que se busca no es un cambio hacia la homogeneización sino el rescate de la diversidad; es decir, partir de las particularidades pero también trabajar sobre ellas y entender los fenómenos socioculturales que rodean a los grupos. Por esto, la importancia trascendental de una indagación en las formas de expresión y comunicación de quienes buscan modificar la forma en que son percibidos. Se busca, entonces, la comunicación para el cambio social como herramienta que transforma agentes culturales y consolida la justicia e igualdad.

Este es un tipo de comunicación totalmente honesta y directa que busca concientizar a diversos públicos sobre una situación, que por su cotidianidad, pasa desapercibida. Los objetivos son claros, atravesar las barreras socioculturales impuestas que actúan como obstáculo para una verdadera interacción y comprensión entre grupos que coexisten en un ambiente determinado. Donde es importante acercarse a ambos bandos y conocer su percepción y actitud actual y potencial. No se trata de 
imponer una idea arbitraria sino llegar a consensos visibles entre grupos que permitan una convivencia benéfica mediante un proceso paulatino. La meta es que a futuro los cambios logrados persistan, logrando así una restructuración de una idea en el inconsciente social.

\section{METODOLOGÍA}

En este apartado se describe el proceso metodológico de la investigación. Se eligió trabajar con cuestionarios para realizar un sondeo de opinión. Roberto Hernández Sampieri, Carlos Fernández Collado, María Baptista (2014, p. 217) citan que este instrumento "consiste en un conjunto de preguntas respecto de una o más variables a medir".

Para este instrumento de investigación se utilizaron únicamente preguntas cerradas. Además se estructuraron y ordenaron las preguntas según varios parámetros. Las preguntas del primer bloque tienen carácter demográfico, estas nos ayudan a conocer el perfil de los encuestados. El segundo bloque trata sobre conocimientos, actitudes y prácticas relacionados con los derechos de los grupos GLBTI.

\section{Población investigada}

La población investigada comprende dos institutos de educación superior privados de la ciudad de Quito: la Pontificia Universidad Católica del Ecuador - PUCE y a la Universidad Politécnica
Salesiana - UPS. Según información brindada por las dos universidades el universo corresponde a 10812 estudiantes de la PUCE y a 10911 estudiantes de la UPS, para un total de 21723 individuos, durante el período en que se levantó la información.

\section{Muestra}

Para el caso de la investigación los parámetros para la determinación de la muestra son los siguientes:

- $\quad N=21723$ individuos

- $\quad K=1,96$ el nivel de confianza (este es el valor de K para un nivel de confianza del 95\%, de acuerdo con las tablas de la distribución normal z) $p=0,5$ la probabilidad de éxito para el presente estudio es del 50\%

- $\quad q=0,5$ se obtiene de restar 1-p. El resultado para este caso es del 50\% e $=0,075$ la diferencia que existirá entre el estimador que sale de la muestra - por ejemplo la media de la muestra - y la media de la población 
Por lo tanto, la muestra calculada fue de 161 individuos.

Se determinó la aplicación de 161 encuestas útiles para análisis según conceptos del muestreo no probabilísti$\mathrm{co}$, en el que el investigador escoge a los participantes bajo su criterio, es decir no se obtiene la muestra bajo una fórmula matemática al azar, por lo tanto no todos los sujetos que forman parte de la población tienen la posibilidad de participar en la investigación. Cabe resaltar que los resultados obtenidos mediante este tipo de muestreo no pueden ser generalizados para el universo, sino que poseen validez interna en el entorno estudiado (Blanco, 2011).

\section{Procedimiento muestral}

Se utilizó el muestreo no probabilístico casual o por conveniencia debido a la factibilidad temporal, económica y de acceso a la información de las instituciones educativas con respeto a sus estudiantes y personal administrativo.

\section{Alcance}

Área geográfica que comprende al norte del Distrito Metropolitano de Quito.

\section{Tiempo}

La encuesta se aplicó en el período del 11 de abril al 04 de mayo del 2015.

\section{RESULTADOS Y DISCUSIÓN}

Las tablas No 1, 2 y 3 revelan la información demográfica de las 161 personas que fueron objeto de la encuesta de forma voluntaria. En primer lugar, podemos decir que el 92,6\% de los participantes fueron personas entre 15 y 30 años, lo que representa un porcentaje importante para aproximarnos a los conocimientos, actitudes y prácticas de este grupo, formado tanto por adolescentes como adultos jóvenes muestra hacia la comunidad GLBTI. En cuanto al género, las tablas también muestran que el $40,4 \%$ de los participantes fueron hombres, mientras que la mayoría, el 59,6\% fueron mujeres quienes estuvieron dispuestos a responder preguntas con respecto al tema (tablas 1 y 2 ).

Tabla 1

\begin{tabular}{|c|c|}
\hline Edad & $\mathbf{\%}$ \\
\hline $15-20$ años & 11,8 \\
\hline $21-25$ años & 67,1 \\
\hline 26 - 30 años & 13,7 \\
\hline 31 - 35 años & 3,1 \\
\hline 36 - 40 años & 3,7 \\
\hline más de 40 & 0,6 \\
\hline Total & 100 \\
\hline
\end{tabular}


Tabla 2

\begin{tabular}{|c|c|}
\hline Género & \% \\
\hline Masculino & 40,4 \\
\hline Femenino & 59,6 \\
\hline Total & 100 \\
\hline
\end{tabular}

Finalmente, la tabla 3 muestra que el $89,4 \%$ de los participantes cursan la educación superior, un 8,1 \% estudia la secundaria y el 2,5\% tienen formación de posgrado. Por lo tanto, el $100 \%$ de los participantes de la encuesta son personas formadas académicamente, pues han recibido entre 8 y 19 años de educación aproximadamente.

Tabla 3

\begin{tabular}{|l|c|}
\hline Nivel educativo & \% \\
\hline Primaria & 0 \\
\hline Secundaria & 8,1 \\
\hline Superior & 89,4 \\
\hline Posgrado & 2,5 \\
\hline Otro & 0 \\
\hline Total & 100 \\
\hline
\end{tabular}

Según la tabla 4, casi las tres cuartas partes de los encuestados respondieron afirmativamente conocer lo que GLBTI quiere decir, mientras que el resto de ellos respondió que no. Pero para comprobar los conocimientos reales de dicha respuesta, se realizó la siguiente pregunta abierta de comprobación: Si su respuesta fue afirmativa dígame qué es GLBTI en sus palabras. Mediante esta se confirmó que menos de la mitad de aquellos que respondieron que sí, realmente sabían el significado correspondiente de las cinco letras que involucra el término (Tabla 5).

Tabla 4

\begin{tabular}{|l|c|}
\hline \multicolumn{2}{|l|}{ ¿Conoce usted qué quiere decir GLBTI? } \\
\hline Respuesta & $\%$ \\
\hline Sí conoce & 72 \\
\hline No conoce & 28 \\
\hline Total & 100 \\
\hline
\end{tabular}

Es importante recalcar que se presenta mayor dificultad para señalar las palabras correspondientes a las siglas $T$ e I, que se refieren a personas Trans e Intersexuales respectivamente. Además se visibiliza una gran dificultad entre lo que se denominan personas trans, ya que se utilizan los términos: travesti, transexual y transgénero como sinónimos y sin ninguna distinción particular. Como se puede observar en la tabla 5 no todas las personas supieron expresar el significado total del acrónimo. Cerca de una cuarta parte que respondió 4 de 5 letras de forma correcta en general, no mencionaron la palabra intersexual. Mientras que en el $11 \%$ se encontraban las personas que no manifestaron el significado de la T ni la letra I. 
Tabla 5

\begin{tabular}{|l|c|}
\hline Significado de las siglas GLBTI & \% \\
\hline 5 correctas & 43 \\
\hline 4 correctas & 23 \\
\hline 3 correctas o menos & 11 \\
\hline Otras & 23 \\
\hline Total & 100 \\
\hline
\end{tabular}

Aclaremos el significado de los términos que generan mayor confusión. Se considera travesti a aquella persona que utiliza actitudes y prendas de vestir que se conciben como propios del sexo opuesto. Un transgénero es una persona cuya concepción de su género no corresponde al de su sexo biológico, existen transgéneros femeninos y masculinos. Mientras que un transexual es aquel que recurre a modificaciones mediante cirugías para cambiar su sexo. Por último, se denomina intersexual a personas que poseen órganos genitales de ambos sexos (INEC, 2013).

Entonces podríamos decir que se mantiene una concepción vaga del término, ya que se tiene una referencia general hacia la homosexualidad. Esta generalidad dejaría fuera a la diversidad de grupos sexuales que se trata de representar. Pero recordemos un hecho importante "antes del año 1997 la palabra homosexual se utilizaba para referir a cualquier persona con una orientación sexual distinta a la heterosexual" (Quinta- na Zurita, 2014, p. 31). Esto podría sugerir que la generalidad que persiste sobre lo asociado con lo GLBTI se debe a que una carga histórica y cultural permanece arraigada en el imaginario de nuestra sociedad y aun con el paso de los años no se logra superar, lo que conlleva a una falta de posicionamiento sobre las nuevas concepciones sobre la sexualidad en general.

Según los datos arrojados por el sondeo (Tabla 6), el 95 \% de los encuestados de la muestra cree que en Ecuador existe discriminación hacia los homosexuales. Este resultado corresponde con la percepción de los grupos GLBTI del país. Según un estudio del INEC (2013) reveló que el 70,9\% reportó haber tenido experiencias de discriminación en su entorno familiar. Este dato refleja una percepción parecida en los públicos ya que los estudios muestran cifras que corresponden a un alto grado de vulneración de los derechos de las personas de esta comunidad.

Tabla 6

\begin{tabular}{|l|r|}
\hline \multicolumn{2}{|l|}{$\begin{array}{l}\text { ¿Cree usted que en Ecuador } \\
\text { existe discriminación hacia los } \\
\text { homosexuales? }\end{array}$} \\
\hline Si & $95 \%$ \\
\hline No & $5 \%$ \\
\hline Total & $100 \%$ \\
\hline
\end{tabular}


Analicemos al público que respondió que no cree que exista discriminación en el país. Primero, su rango de edad está entre los 15 y los 25 años, todos ellos tienen un nivel educativo superior con una excepción. Se esperaría que quienes respondieran de forma negativa a la pregunta tuvieran una edad superior a los 30 años pues el concepto de discriminación por orientación sexual es relativamente nuevo en nuestro medio. Y las prácticas de exclusión y rechazo hacia los grupos GLBTI que se daban anteriormente, podrían haber sido consideradas como formas comunes de trato, mas no vistas como acciones que excluían a un grupo humano pues ni siquiera se contemplaba sus derechos como grupo en la normativa legal.

Para mostrar cómo la población percibe la agresión hacia los grupos GLBTI se realizaron varias preguntas, entre las respuestas encontramos que el $57 \%$ de los encuestados afirmó haber sido testigo de un acto discriminatorio o violento hacia personas con preferencia sexual diversa. Dentro de este grupo respondieron que las formas más comunes de agresión presenciadas fueron: en un $40 \%$ amenazas, $39 \%$ insultos, $13 \%$ agresión física y $8 \%$ burlas. Sin duda las muestras de agresión verbal están más presentes que las físicas pero ¿por qué dichas prácticas parecen ser toleradas por la sociedad? (Tablas 7 y 8 )
Tabla 7

\begin{tabular}{l|r|}
\hline \multicolumn{2}{|l|}{ ¿Ha presenciado algún hecho de } \\
violencia o discriminación contra los \\
grupos GLBTI?
\end{tabular}

Tabla 8

\begin{tabular}{|l|r|}
\hline \multicolumn{2}{|l|}{$\begin{array}{l}\text { Formas de agresión presenciadas } \\
\text { hacia grupos GLBTI }\end{array}$} \\
\hline Amenazas & $40 \%$ \\
\hline Insultos & $39 \%$ \\
\hline Burlas & $13 \%$ \\
\hline Agresión física & $8 \%$ \\
\hline Total & $100 \%$ \\
\hline
\end{tabular}

Al parecer estas ideas de violencia legitimada desembocan de actitudes y prácticas que las personas han vivido en su infancia. Soria (2015) se refiere a la percepción de las agresiones en diferentes niveles, lo que simbolizan los términos despectivos, la agresión verbal y las actitudes que fomentan las manifestaciones de violencia contra los grupos GLBTI. Por ejemplo señala que: "Mucha gente no sabe cómo utiliza a diario los términos que pueden ser muy peyorativos. Es una falta de conciencia: el papá que le dice al hijo cuando se cae "calle mijito no sea maricón", le está enseñando al chico a ser homofóbico". 
Esta aseveración es totalmente válida, por ejemplo, Núñez Noriega (2011, p. 87) señala en su libro que:

[e]sta violencia contra todos los varones utiliza frecuentemente como estrategia pedagógica y como producto mismo de la lógica cultural homofóbica, la violencia física y emocional hacia los niños, los púberes y los adolescentes considerados débiles, afeminados o que no son suficientemente masculinos, según los parámetros de género. La violencia contra los afeminados o menos hombres o que en algún momento específico fallaron en el cumplimiento del ideal masculino, se convierte así en un ejemplo elocuente para los otros niños, hermanos o conocidos; un ejercicio público de violencia socialmente tolerado que envía un mensaje de amenaza para quienes se atrevan a transgredir el orden de las identidades de género(....."

Parece ser que una de las principales causas de la aceptación de este tipo de violencia es la falta de conciencia sobre los términos que empleamos a diario y sus implicaciones en distintos ámbitos. Como mencionó Soria, la palabra maricón es un término utilizado para referirse a los homosexuales de manera despectiva, a comportamientos no varo- niles o meramente como insulto vulgar. Además, las actitudes coercitivas que permitimos que se den en los niños si estos no muestran un comportamiento adecuado según su sexo parecen ser ideas que cimientan la discriminación desde temprana edad.

La tabla 9 también está relacionada con la segregación de un grupo social, pues a la pregunta: ¿Usted ha discriminado a alguna persona de la comunidad GLBTI? El 91 \% respondió que no pero un $9 \%$ que sí. Aunque el porcentaje de quienes aceptaron haber discriminado es bajo, creemos que por la naturaleza de la pregunta varias personas pudieron no ser sinceras con su respuesta aunque el cuestionario fuera anónimo. Retomemos el estudio sobre grupos GLBTI realizado por el INEC (2013) donde se señala que solo en el ámbito familiar: "El 70,9\% reportó que vivieron alguna experiencia en su entorno familiar de los cuales el 72,1 \% sufrió algún tipo de experiencia de control, el 74,1 \% experimentó algún tipo de imposición, el 65,9\% sufrió algún tipo de rechazo y el 61,4\% de violencia".

\section{Tabla 9}

\begin{tabular}{|l|l|}
\hline \multicolumn{2}{|l|}{ ¿Usted ha discriminado a alguna } \\
persona de la comunidad GLBTI?
\end{tabular}


Diario El Telégrafo (2013) sobre el mismo estudio señala que el $61,4 \%$ de la muestra se ha sentido discriminada, además un 65,9\% ha experimentado rechazo social. Esta realidad señala que un gran porcentaje de personas de la población GLBTI son discriminadas y rechazadas por la demostración pública de su orientación e identidad sexual. Pero por supuesto esto no ocurre solo en el ámbito familiar sino que traspasa a lo público cuando la segregación se da en instituciones educativas, lugar de trabajo, atención médica, calles, plazas, etc.

Para analizar el rol que los medios de comunicación del país cumplen con respecto a los grupos sociales, en este caso la comunidad GLBTI, preguntamos al público su percepción y estas fueron sus respuestas. El $83 \%$ manifestó que no creen que los medios masivos de comunicación difundan los derechos de las personas GLBTI. Además, el 65 \% está de acuerdo en que el contenido que se transmite sobre el grupo mencionado corresponde a notas amarillistas y de crónica roja. Además, una mayoría (55\%) afirma que nunca o casi nunca, en los medios de comunicación se transmite noticias que involucren a grupos GLBTI (Tablas 10, 11 y 12).
Tabla 10

\begin{tabular}{|c|c|}
\hline \multicolumn{2}{|c|}{$\begin{array}{l}\text { ¿Cree usted que los medios de comu- } \\
\text { nicación difunden los derechos de los } \\
\text { grupos GLBTI? }\end{array}$} \\
\hline Sí & $17 \%$ \\
\hline No & $83 \%$ \\
\hline Total & $100 \%$ \\
\hline
\end{tabular}

Tabla 11

\begin{tabular}{|c|c|}
\hline \multicolumn{2}{|c|}{$\begin{array}{l}\text { ¿Cree que el tratamiento que los } \\
\text { medios de comunicación dan a la } \\
\text { información de los grupos GLBTI es } \\
\text { amarillista y de crónica roja? }\end{array}$} \\
\hline Sí & $65 \%$ \\
\hline No & $35 \%$ \\
\hline Total & $100 \%$ \\
\hline
\end{tabular}

Tabla 12

\begin{tabular}{|l|l|}
\hline $\begin{array}{l}\text { ¿Qué tan a menudo ve usted que } \\
\text { en los medios de comunicación se } \\
\text { trasmitan noticias que involucren a } \\
\text { grupos GLBTI? }\end{array}$ \\
\hline Siempre & $1 \%$ \\
\hline Casi siempre & $5 \%$ \\
\hline A veces & $39 \%$ \\
\hline Casi nunca & $51 \%$ \\
\hline Nunca & $4 \%$ \\
\hline Total & $100 \%$ \\
\hline
\end{tabular}


Analicemos estudios y opiniones que nos ayuden a comprender la conducta de los medios de comunicación. El estudio de Igualdad, diversidad y discriminación en los medios de comunicación llevado a cabo por el Ministerio de Inclusión Social y Económica - MIES (2014, p. 30) señala que:

Es frecuente que los medios de comunicación reproduzcan discursos que condenan a las personas GLBTI. Si bien existen excepciones, la tendencia general de los medios es construir en los imaginarios ciudadanos las trans, lesbo y homofobias. Esta recurrencia refleja, que por lo general, las opciones de preferencia sexual son tratadas con claro y reiterado discrimen que contiene cargas machistas y sexistas.(...) A las preferencias de este orden se las suele referir como patologías o perversidades, sugiriendo acciones que corrijan los supuestos defectos a los que se relaciona con conductas peligrosas.

También, en la opinión de la Antropóloga Rocío Rosero (2015):

Hay todavía una tendencia bastante amarillista para tratar los temas de la diversidad sexual. Todavía los contenidos de muchos de los programas que tienen un alto rating tienen que ver con la ridiculización de la identidad sexual, la identidad de género, la condición étnica... El Combo Amarillo y este tipo de programas que tienen como base los hechos discriminatorios, burlescos de la peor estirpe que justamente están fomentando una cultura racista, sexista, homofóbica, que ridiculiza a todos.

Esto nos lleva a pensar que los medios sobreponen el entretenimiento burdo a la información, no solo en programas informativos como noticieros sino en toda la parrilla de programación, si tomamos a la televisión como ejemplo. Y de igual forma en el resto de medios masivos que no dan espacio a temas como la divulgación de los derechos de un grupo social que ha sido constantemente vulnerado. Así también lo señalan los encuestados pues el 51 \% respondió que casi nunca ven la transmisión de noticias que involucren a los grupos GLBTI, un $39 \%$ respondió a veces y un $4 \%$ dijo que nunca. Estos datos indican una situación particular no solo por la poca visibilización que se les da sino la manera en que se los muestra socialmente como sujetos secundarios que ocupan espacios como información de relleno, espectáculo, crónica roja, chismes, etc. Efraín Soria (2015) está de acuerdo con este postulado y desde su punto de vista: 
Los medios de comunicación, en el caso del Ecuador, todavía tienen deudas pendientes con respecto a cómo tratar ciertos temas. Todos los temas que tienen que ver con la sexualidad en general no son bien llevados. Es decir, como son vistas las mujeres, las prácticas sexuales (...), las orientaciones sexuales en este caso las no heterosexuales como son vistas. Entonces, en ese sentido los medios necesitarían educarse.

Nos estamos enfrentando a un patrón cultural arraigado a nuestro contexto y a formas estereotipadas de referirse a las personas que continúan di- fundiéndose a través de los medios cuya función debería ser desmitificarlos, y a pesar que Ecuador cuenta con la Ley Orgánica de Comunicación (2013) que en su Artículo 61 señala la prohibición de emitir contenido discriminatorio, incluyendo la discriminación por orientación sexual.

Si bien se han sentado precedentes de sanción ${ }^{2}$ existe una falta de regulación del contenido de los distintos canales que a manera de entretenimiento continúan con una práctica que genera un concepción equivocada de la diversidad sexual, donde no existe tolerancia ni respeto hacia otros seres humanos cuyos derechos deberían ser garantizados.

\section{CONCLUSIONES}

El público encuestado reflejó una falta de conocimiento generalizado y percepciones vagas sobre conceptos que se refieren a la diversidad sexual, una minoría (apenas el $12 \%$ de la muestra) conoce el significado completo de la sigla GLBTI. Mientras un $60 \%$ muestra un conocimiento parcial y un $28 \%$ no reconoce la sigla.
El público percibe que los medios de comunicación tradicionales en el país emiten material informativo escaso sobre diversidad sexual y derechos de personas GLBTI, pues la mayor parte del tiempo estos temas se distorsionan mediante contenido con carácter amarillista, de farándula, crónica roja o entretenimiento satírico. Solo un $17 \%$ de los

\footnotetext{
En el 2014, la serie de televisión ecuatoriana "La Pareja Feliz" recibió sanciones económicas de la Supercom por tratar contenido discriminatorio por razones de orientación sexual, sexista y machista en varias ocasiones. Teleamazonas, canal por el cual se transmitía el programa a nivel nacional, decidió finalmente sacarla del aire (Diario El Comercio, 2014).
} 
encuestados opinó que los medios de comunicación difunden los derechos de los grupos GLBTI, mientras que un 51\% expresó que se trasmiten noticias que involucren a grupos GLBTI. Lo que evidencia un manejo pobre y poco interesado en temas que se relacionan con la sexualidad. Por consiguiente, los medios de comunicación permanecen como actores que no contribuyen a una visibilización adecuada, sino superficial sobre la problemática.

Además, la muestra de jóvenes universitarios encuestada percibe un ni- vel alto de discriminación y violencia hacia este colectivo, lo que evidencia que el cambio social aún no se ha producido en nuestro medio. Se puede presumir, entonces, que estas manifestaciones ilegales e ilegítimas, aún se encuentran presentes, de forma constante, en la esfera pública.

Finalmente, se puede concluir que la falta de conocimiento de las siglas GLBTI supone, además, un problema de invisibilización de los grupos, debido, al parecer, a que están marginalizados del proceso reflexivo universitario. 


\section{REFERENCIAS}

Asamblea Nacional. (2013). Ley Orgánica de Comunicación. Quito: Editora Nacional.

Blanco, C. (2011). Encuesta y Estadística. Métodos de Investigación Cuantitativa en Ciencias Sociales y Comunicación. Argentina: Editorial Brujas.

De Fleur, M. (1972). Teoría de la comunicación masiva. Buenos Aires: Paidos.

Diario El Comercio. (17 de noviembre de 2014). La 'Pareja feliz' ya no se transmitirá. Obtenido de http:// www.elcomercio.com/tendencias/parejafeliz-transmision-television-sancion-supercom.html

Diario El Telégrafo. (10 de diciembre de 2013). Encuesta del INEC muestra que aún hay discrimen hacia la comunidad gay. Recuperado el 18 de mayo de 2015, de Diario El Telégrafo: http://www.telegrafo.com.ec/sociedad/item/ el-60-de-glbti-se-siente-discriminado-en-los-espacios-publicos. html

Fernández Collado, C., \& Galguera García, L. (2008). La comunicación humana en el mundo contemporáneo. México: McGraw- Hill.

Garrido, A., \& Álvaro, J. L. (2007). Psicología Social. Perspectivas psicológicas y sociológicas. Madrid: McGraw Hill.
Gumucio, A. (2004). Pontificia Universidad Javeriana de Bogotá. Obtenido de http://www.javeriana.edu.co/ documents/245769/3062963/ CUARTO+MOSQUETERO+GUMUCIO.pdf/3b954ba4-1f95-4ea885be-830a65a2721f

Hernández Sampieri, R., Fernández Collado, C., \& Baptista Lucio, P. (2014). Metodología de la Investigación. México: Mc Graw Hill.

INEC. (2013). Estudio de caso sobre condiciones de vida, inclusión social y cumplimiento de Derechos Humanos de la población GLBTI en Ecuador. Quito. Obtenido de http:// www.ecuadorencifras.gob.ec/ documentos/web-inec/Estadisticas_Sociales/LGBTI/Analisis_situacion_LGBTI.pdf

Ministerio de Inclusión Económica y Social - MIES. (2014). Igualdad, diversidad y discriminación en los medios de comunicación. Obtenido de MIES: http://www. inclusion.gob.ec/wp-content/ uploads/downloads/2014/04/LIBRO-Igualdad-Medios-web-vale. pdf

Núñez Noriega, G. (2011). ¿Qué es la diversidad sexual? Quito: Abya-Yala.

O'Sullivan, T., Hartley, J., Saunders, D., 
Montgomery, M., \& Fiske, J. (1997). Rosero Garcés, R. (3 de Mayo de 2015). Conceptos clave en comunicación Percepción social de los Derechos y estudios culturales. Buenos Aires: Amorrortu editores. de los grupos GLBTI. (F. Cadena, Entrevistador).

Pacheco, F. (2014). Procedimiento Muestral.[ Material de clase]. Pontificia Universidad Católica del Ecuador. Quito. E. (3 de 09 de 2015). Desarrollo de las campañas de la Fundación Equidad. (F. Cadena, Entrevistador).

Quintana Zurita, Y. (2014). Balance y perspectivas de los Derechos Humanos de las personas LGBTI en el Ecuador. Quito: El Telégrafo. 86. 